\title{
VULNERABILIDAD LABORAL DE LA MUJER RURAL LATINOAMERICANA
}

\author{
Labor vulnerability of Latin \\ American rural women
}

Jaime de Pablo Valenciano ${ }^{1}$, Mercedes Capobianco Urdiales ${ }^{2}$, Juan Uribe Toril ${ }^{3}$

Fecha de recepción: 14 de marzo de 2016.

Fecha de aceptación: 20 de junio de 2016

\begin{abstract}
1- Nacionalidad: Española. Grado: Doctorado en Ciencias Económicas. Especialización: Economía aplicada, Economía agraria. Procesos de integración. Adscripción: Universidad de Almería (España). Correo: jdepablo@ual.es

2- Nacionalidad: Española. Grado: Master en Prevención de Riesgos Laborales. Especialización: Ingeniera Biotecnológica. Diplomado en Ciencias Empresariales. Adscripción: Universidad de Almería (España). Correo: mercedescapobianco@hotmail.com
\end{abstract}

3- Nacionalidad: Española. Grado: Doctorado en Ciencias Económicas y Empresariales por la Universidad de Almería. Especialización: Economía y empresa. Economía aplicada. Administración de proyectos. Adscripción: Profesor Asociado de la Universidad de Almería (España). Gerente de la Fundación Andalucía-Emprende en Almería. Correo: juribe@ual.es 


\section{Resumen}

Este artículo aborda el estudio de la situación laboral de las mujeres rurales en los países latinoamericanos. Las mujeres rurales son uno de los grupos sociales más vulnerables en el ámbito laboral latinoamericano, debido a su baja participación laboral, a la categorización ocupacional mayoritaria como trabajadoras por cuenta propia y familiar no remunerado y a índices de feminización positivos. Pero sus posibilidades de mejorar su condición de vulnerabilidad social laboral se centra principalmente en el Empleo Rural No Agrario (ERNA), con gran potencial en el desarrollo rural, y al apoyo legislativo en cuanto al acceso a los bienes de tierra. Frente al proceso de urbanización es necesario implementar políticas públicas que tengan en cuenta la existencia de las mujeres rurales como trabajadoras en el ámbito rural y diseñar medidas de ayuda especialmente dirigidas a este colectivo.

Palabras claves: empoderamiento, propiedad de la tierra, políticas públicas.

\section{Abstract}

This article deals with the study of the labor situation of rural women in Latin American countries. Rural women are one of the most vulnerable social groups in the Latin American workplace, due to its low labor participation, occupational majority self categorization as workers and unpaid family account and positive rates of feminization. But their chances of improving their social vulnerability status of work focuses primarily on nonagricultural Rural Employment (NARE), with great potential in rural development, and legislative support in access to land assets. Opposite the urbanization process is necessary to implement public policies that take into account the existence of rural women as workers in rural areas and design support measures specifically aimed at this group.

Keywords: empowerment, land ownership, public policy. 


\section{Introducción}

$\mathrm{D}$ esde el 1 de enero de 2016, entraron en vigencia los 17 objetivos de desarrollo sostenible (ODS) para el periodo 2016-2030, los cuales, en parte, reemplazan a los objetivos del milenio del periodo 2000-2015. El secretario general de la ONU, Ban Ki-moon (2016), manifestó que los nuevos Objetivos de Desarrollo Sostenible (ODS) tienen en su centro las cuestiones de la igualdad de género y el empoderamiento de la mujer, e incluyen una meta para 'duplicar la productividad agrícola y los ingresos de los productores de alimentos en pequeña escala, en particular las mujeres'. En efecto, las mujeres rurales son fundamentales para el éxito de la casi totalidad de los 17 ODS. Las mujeres rurales son agentes clave para conseguir los cambios económicos, ambientales y sociales necesarios para el desarrollo sostenible pero su acceso limitado al crédito, la asistencia sanitaria y la educación son algunos de los muchos retos a los que se enfrentan. Estos se ven agravados además por las crisis mundiales - económica y alimentaria - y el cambio climático. Garantizar su empoderamiento no sólo es fundamental para el bienestar de las personas, familias y comunidades rurales, sino también para la productividad económica general, dada la amplia presencia de mujeres en la mano de obra agrícola mundial.

La Organización de las Naciones Unidas Mujeres (ONU Mujeres, 2015) sostiene sobre el empoderamiento de las mujeres rurales a través de los ODS que las agricultoras controlan menos tierra que los hombres y tienen un acceso limitado a los insumos, las semillas, el crédito y los servicios de extensión. Menos del 20 por ciento de los propietarios de tierras son mujeres. Las diferencias de género en el acceso a la tierra y el crédito influyen en la capacidad relativa de las agricultoras y los agricultores y las personas emprendedoras para invertir, operar a escala, y beneficiarse de nuevas oportunidades económicas.

Históricamente, las zonas rurales se caracterizan por estructuras de poder y costumbres sociales que cambian lentamente. Todo ello conlleva a una situación de vulnerabilidad a las mujeres rurales, sufriendo una doble marginación: por ser mujeres y por ser rurales (Quintanilla Barba, 2002:1).

El objetivo de este artículo es conocer la situación de vulnerabilidad social en la que se encuentran el colectivo de mujeres rurales latinoamericanas en el ámbito laboral e indicar medidas relacionadas con políticas públicas que sirvan para salir de la situación presente.

Inicialmente se tratarán los conceptos de ruralidad y los aspectos conceptuales sobre vulnerabilidad, vulnerabilidad social y vulnerabilidad social laboral. A continuación se estudiará la distribución de mujeres rurales latinoamericana por países y un análisis del trabajo rural femenino. Finalmente se estudiará la distribución de la propiedad de tierras, la evolución temporal de la legislación latinoamericana al respecto segregada por sexo y estado civil. Se concluirá con orientaciones para el desarrollo de políticas gubernamentales para mejorar la situación de vulnerabilidad de la mujer rural trabajadora latinoamericana.

\section{Aspectos conceptuales de ruralidad y vulnerabilidad en el mercado laboral}

A nivel mundial no existe un concepto universal de ruralidad, habiendo en la actualidad diversos criterios para definirla (Faiguenbaum, 2011:67). En América Latina, la definición de ruralidad varía según el país y además se han ido modificando a lo largo de los años. En el Cuadro 1 se puede observar esta diversidad de definiciones sobre áreas rurales por país, combinando los distintos criterios existentes con la aplicación de diversas condiciones. 


\section{Cuadro 1. Criterios y condiciones utilizados en definiciones} de zona rural de países latinoamericanos

\begin{tabular}{|c|c|c|}
\hline Criterios & Condiciones & Países \\
\hline \multicolumn{3}{|c|}{ Demográfico } \\
\hline \multirow{3}{*}{ Cantidad de habitantes } & Menos de 1.500 & Panamá \\
\hline & Menos de 2.000 & Bolivia, Argentina \\
\hline & Menos de 2.500 & Venezuela, México \\
\hline \multicolumn{3}{|c|}{ Demográfico y económico } \\
\hline \multirow{2}{*}{$\begin{array}{l}\text { Cantidad de habitantes y } \\
\text { ocupación de la población } \\
\text { económicamente activa }\end{array}$} & Menos de 1.0000 & \multirow[b]{2}{*}{ Chile } \\
\hline & $\begin{array}{l}\text { menos de } 2.000 \text { con más del } 50 \% \text { de la } \\
\text { PEA dedicada a actividades primarias }\end{array}$ & \\
\hline \multicolumn{3}{|c|}{ Demográfico y funcional } \\
\hline \multirow{6}{*}{$\begin{array}{l}\text { Cantidad de habitantes y } \\
\text { servicios }\end{array}$} & Menos de 500 o & \multirow[b]{2}{*}{ Cuba } \\
\hline & $\begin{array}{l}\text { entre 501-2.000 con menos de } 4 \text { servi- } \\
\text { cios urbanos }\end{array}$ & \\
\hline & Menos de 2.0000 & \multirow[b]{2}{*}{ Guatemala } \\
\hline & $\begin{array}{l}\text { el } 51 \% \text { de hogares no disponga de } \\
\text { alumbrado eléctrico y servicio de agua } \\
\text { por tuberías }\end{array}$ & \\
\hline & Menos de 1.000 y sin servicios & Nicaragua \\
\hline & Menos de 2.000 y sin servicios & Honduras \\
\hline \multicolumn{3}{|c|}{ Administrativo } \\
\hline \multicolumn{2}{|c|}{ Residencia fuera de áreas urbanas } & $\begin{array}{l}\text { osta Rica, Brasil, Perú , Colombia, Repú- } \\
\text { minicana, Ecuador, Haití }\end{array}$ \\
\hline \multicolumn{3}{|c|}{ Legal } \\
\hline \multicolumn{2}{|c|}{ Criterios prácticos y de tipo operativos en la legislación } & Uruguay \\
\hline
\end{tabular}

Fuente: Elaboración propia con datos de CEPAL (Sergio Faiguenbaum, 2011:67)

Se puede apreciar en el cuadro anterior las trece definiciones diferentes en los veinte países latinoamericanos. Genera una preocupación a nivel mundial de las agencias internacionales, ya que consideran la necesidad de efectuar comparaciones de datos estadísticos poblacionales segregados por área de residencia entre países en base a criterios homogéneos. Según Sabalain (2011:26) podría obtenerse una definición homogénea combinando la densidad de población más la distancia a centros poblados se pueda dar cuenta de las diferencias entre países grandes y pequeños, densamente poblados o dispersos. No resulta del todo sencillo intentar una redefinición que dé respuesta a las tres clases de inquietudes debatidas: comparabilidad entre países, dinámica de la ruralidad, mayor relevancia para las políticas.

La definición de ruralidad es relevante para estudios sobre mujer rural porque se identifica por su lugar de residencia en zonas rurales. Pero en el caso de los países latinoamericanos es complicado debido a que utilizan diversos criterios para diferenciar las zonas rurales de las urbanas y no es posible identificar a las mujeres rurales de una manera única.

La vulnerabilidad social consiste en el desajuste entre los activos y la estructura de oportunidades, los activos serían insuficientes, poco pertinentes o difíciles de manejar para aprovechar la estructura de oportunidades existentes (Rodríguez, 2001:18). Los activos pueden consistir en patrimonio físico, ahorros financieros, capital humano y social 
(Weller, 2009:7) y las estructuras de oportunidades como probabilidades de acceso a bienes, servicios o actividades que inciden sobre el bienestar del hogar porque le facilitan el uso de recursos propios o le suministran recursos nuevos, útiles para la movilidad e integración social a través de los canales existentes (Kaztman, 2000:299).

En la población latinoamericana, los problemas estructurales de inserción laboral constituyen un componente clave de la vulnerabilidad social que se puede caracterizar como laboral. Ella no afecta a un grupo por azar, y se puede comprobar analizando el mercado laboral donde determinados grupos muestran peores indicadores que otros. La vulnerabilidad laboral se centra en esos grupos en la falta de acceso a actividades y oportunidades laborales que les facilitarían los ingresos para mejorar significativamente sus condiciones de vida (Weller, 2009:8). Diferentes colectivos de la población en edad de trabajar muestran distintos grados de vulnerabilidad frente al riesgo de caer en alguna de las formas de exclusión laboral, por lo que no sólo es importante identificar los indicadores relevantes para cada una de éstas, sino también analizarlos de manera diferenciada según características como sexo, educación, edad, etnia y entorno territorial (zonas urbanas versus rurales, áreas en expansión productiva versus zonas estancadas) (Weller, 2012:85). La vulnerabilidad hace alusión directa a la incapacidad de los grupos más débiles de la sociedad para enfrentar, neutralizar u obtener beneficios de los impactos provocados por eventos económicos y/o sociales sobre los modelos de desarrollo imperantes, tal que éstos los llevan a un estado de exclusión, no sólo social sino que económica, política y legal (Pizarro, 2001:7).

\section{La mujer rural latinoamericana en el ámbito laboral}

\subsection{La mujer rural latinoamericana}

Actualmente, como se indica en el cuadro 2, la población latinoamericana asciende a más de 600 millones de personas, de las cuales aproximadamente 121 millones habita en zonas rurales, suponiendo más de un $20 \%$ de la población total. El colectivo de mujeres rurales en América Latina supera los 58 millones, representando el 9,72\% de la población total latinoamericana. '

Cuadro 2. Población latinoamericana segregada por sexo y área de residencia. 2013 (miles de personas)

\begin{tabular}{|c|c|c|c|}
\hline & Urbana & Rural & total \\
\hline Mujeres & 247.961 & 58.702 & 306.663 \\
\hline Hombres & 234.684 & 62.351 & 297.035 \\
\hline ambos sexos & 482.645 & 121.053 & 603.698 \\
\hline
\end{tabular}

Fuente: Elaboración propia a través de interpolación de datos 2010 y proyecciones poblacionales 2015 de URPAS2013 (Urban and rural population by age and sex) de la División de Población del Departamento de Economía y asuntos sociales de las Naciones Unidas

En el cuadro 2 se puede observar que la proporción de las mujeres es menor que la de los hombres en las zonas rurales, pero mayor en las urbanas. Según el informe de Oxfam (2014), dependiendo de los territorios que habitan, de su situación económica y de la condición de género, hombres y mujeres viven estos impactos de manera diferenciada. 
De los efectos a corto plazo del cambio climático que las mujeres campesinas reportan y han identificado son: la variación en el clima y la dificultad de distinguir las estaciones, impidiéndoles proveer alimentos para sus familias, en muchos casos la desnutrición está presente en sus comunidades. En consecuencia, las mujeres que habitan en espacios rurales se ven forzadas a migrar y a buscar empleo en las ciudades, ya que en el campo no logran tener ingresos suficientes para vivir.

En la gráfica 1 se observa que la proporción de población rural varía sensiblemente según el país. Desde países altamente ruralizados, situados generalmente en América Central, como Guatemala, donde casi la mitad de su población (49,31\%) es rural. Hasta países fuertemente urbanizados, ubicados mayoritariamente en el cono sur, como Uruguay y Argentina, donde la población rural apenas supera el 7\% de la población total. El país más urbanizado es Venezuela, apenas el 6,10\% de la población total reside en áreas rurales. La proporción de población rural en Latinoamérica, corresponde a más de un $20 \%$, encontrándose en un punto medio entre los países fuertemente urbanizados y los altamente ruralizados.

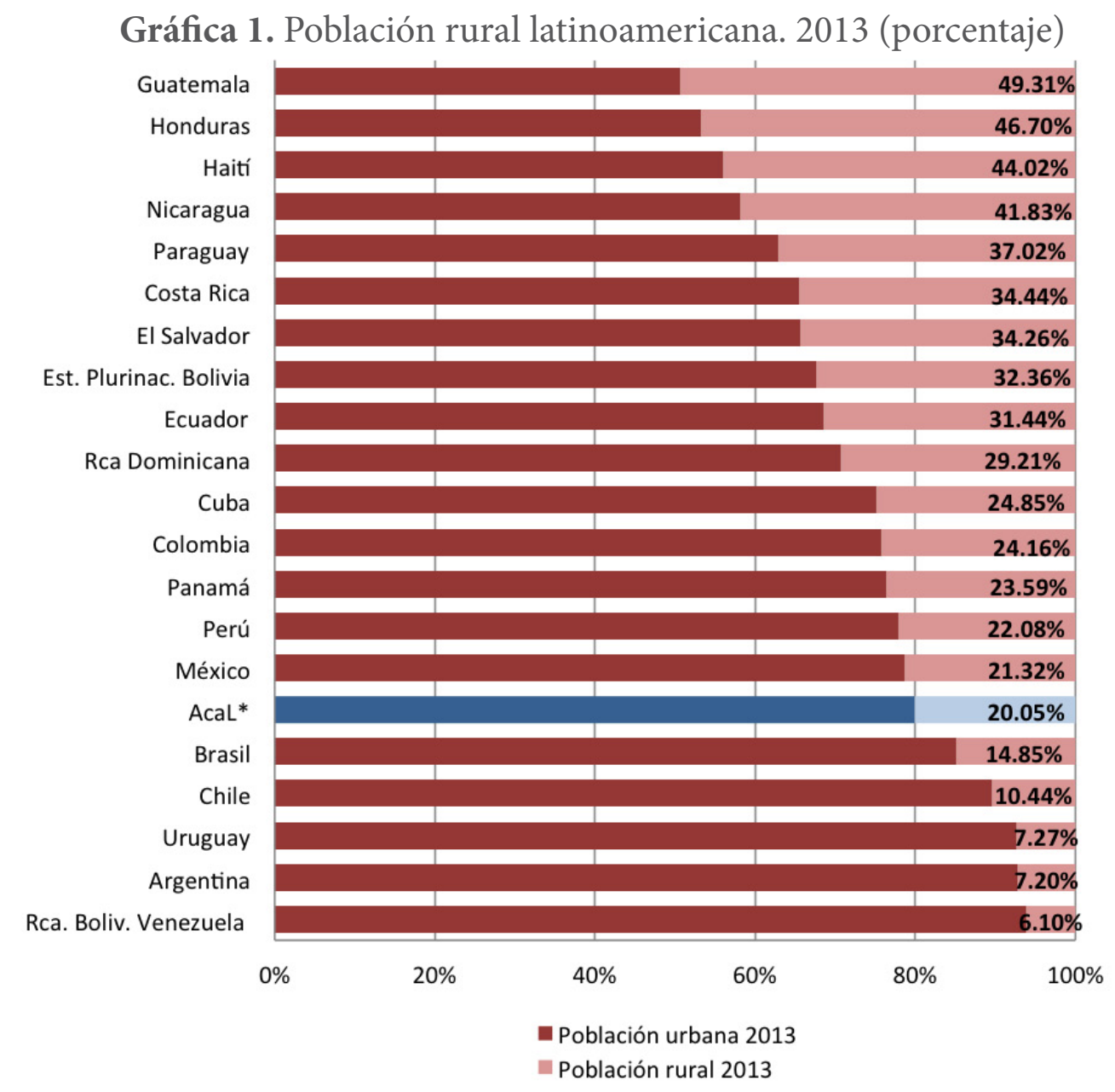

Se puede observar en la gráfica 2 que la distribución de mujeres rurales por países es heterogénea. Los países que presentan mayores porcentajes de mujeres rurales en su población coinciden con los países altamente ruralizados. Es el caso de Guatemala, Honduras, Haití y Nicaragua, donde entre el 20 y 25\% de la población total de estos países son mujeres que residen en zonas rurales. En los países altamente urbanizados la proporción de mujeres rurales desciende a un rango del 3 al $7 \%$. 
- Revista de Ciencias Sociales y Humanidades. ISSN-P: 0188-9834 ISSN-E: 2395-8669.

Gráfica 2. Población rural femenina en América Latina. 2013 (miles de personas)

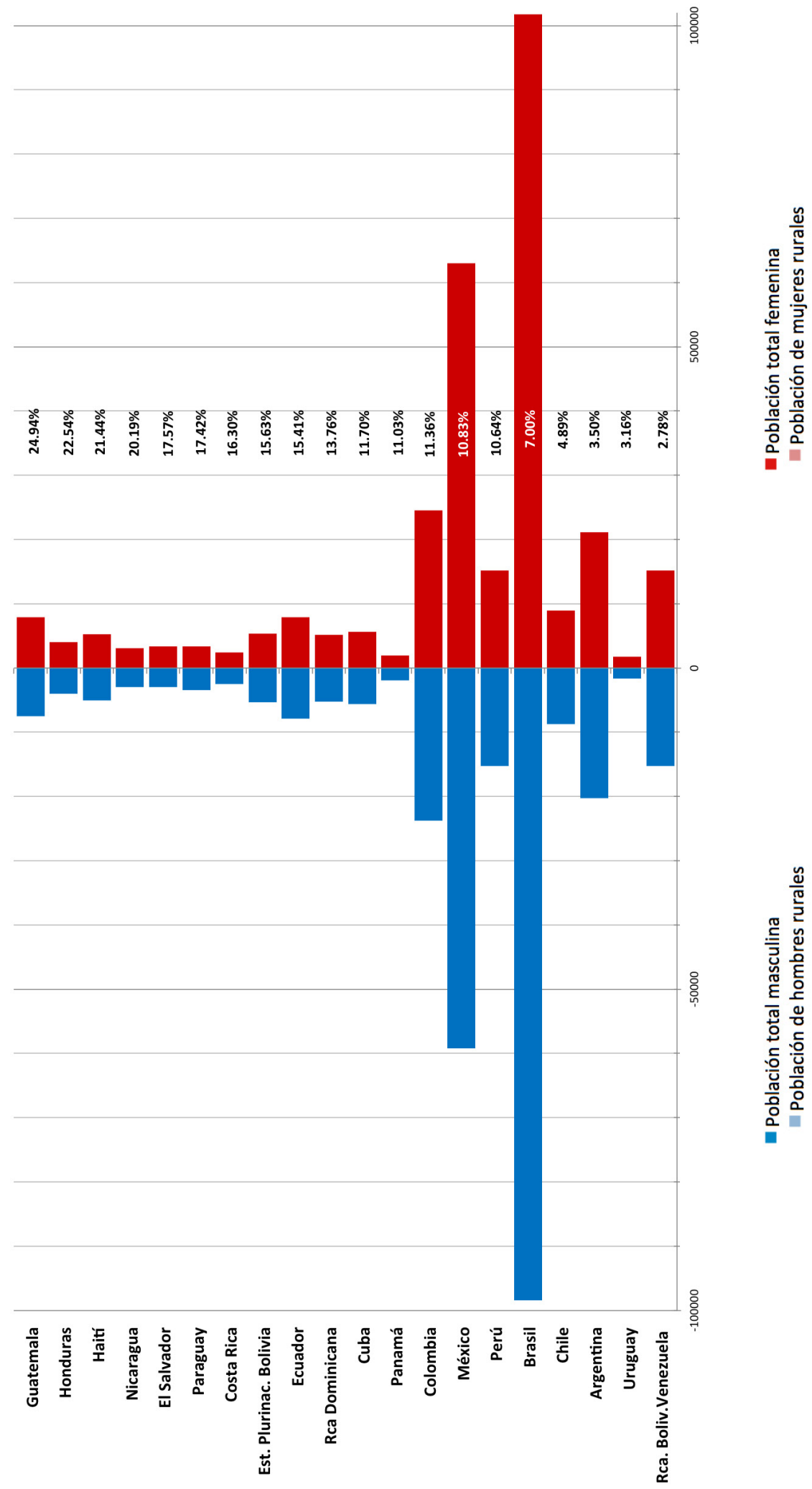


La distribución poblacional rural latinoamericana y en concreto la femenina son heterogéneas. En Latinoamérica están ubicados países altamente urbanizados como la mayoría del Cono Sur (Brasil, Chile, Argentina y Uruguay) frente a otros fuertemente ruralizados (Guatemala, Honduras, Haití, Nicaragua, El Salvador y Costa Rica). En estos países centroamericanos el porcentaje de mujeres rurales es elevado. En promedio, un 21,55\% de la población de estos países son mujeres rurales, pero a nivel de población global son aproximadamente 11 millones de los 600 millones de habitantes en América Latina $(1,82 \%)$ es población femenina rural. Esta distribución poblacional latinoamericana coincide con el panorama planteado por el Fondo de Población de las Naciones Unidas (FPNU). Indica que el proceso de urbanización avanza rápidamente, pero la situación de los países presenta diferencias que condicionan fuertemente el tipo de políticas que se llevan adelante y ha destacado la necesidad de políticas urbanas en un mundo cada vez más urbanizado (FPNU, 2010:95).

Conocer la distribución de ésta población rural femenina es fundamental para la elaboración, puesta en marcha de políticas públicas y distribución de fondos de gobiernos locales y organismos internacionales destinados a este colectivo. FPNU solicita atención especial a la población rural, ya que de otra manera suele ser olvidada en los análisis y las políticas, que se concentran en el ámbito urbano por su creciente predominio demográfico, económico y sociocultural (FPNU, 2010:37).

\subsection{El trabajo rural femenino}

El análisis del trabajo rural femenino conlleva a la necesidad de analizar algunos parámetros económicos que permiten visualizar la realidad que afronta la mujer rural con respecto al hombre rural. Uno de estos parámetros es la tasa de participación laboral femenina.

La tasa de participación femenina es baja, sobre todo en las zonas rurales, para lo cual se conjugan factores como pautas culturales, la ausencia de alternativas para el cuidado y la relativa escasez de oportunidades de empleo remunerado para mujeres (en ocasiones exceptuando empleos temporales), si bien a veces, además, hay problemas de medición que inciden en una subestimación de la participación laboral de las féminas rurales (Weller, 2012:88).

En la gráfica 3 se puede apreciar la tasa específica de participación laboral de la población rural latinoamericana segregada por sexo, donde se puede apreciar que sólo dos países presentan tasas elevadas de participación laboral femenina, Bolivia y Perú (superan el $70 \%$ de la población activa femenina). 
Gráfica 3. Tasa específica de participación laboral de la población rural latinoamericana segregada por sexo. 2013 (porcentajes)

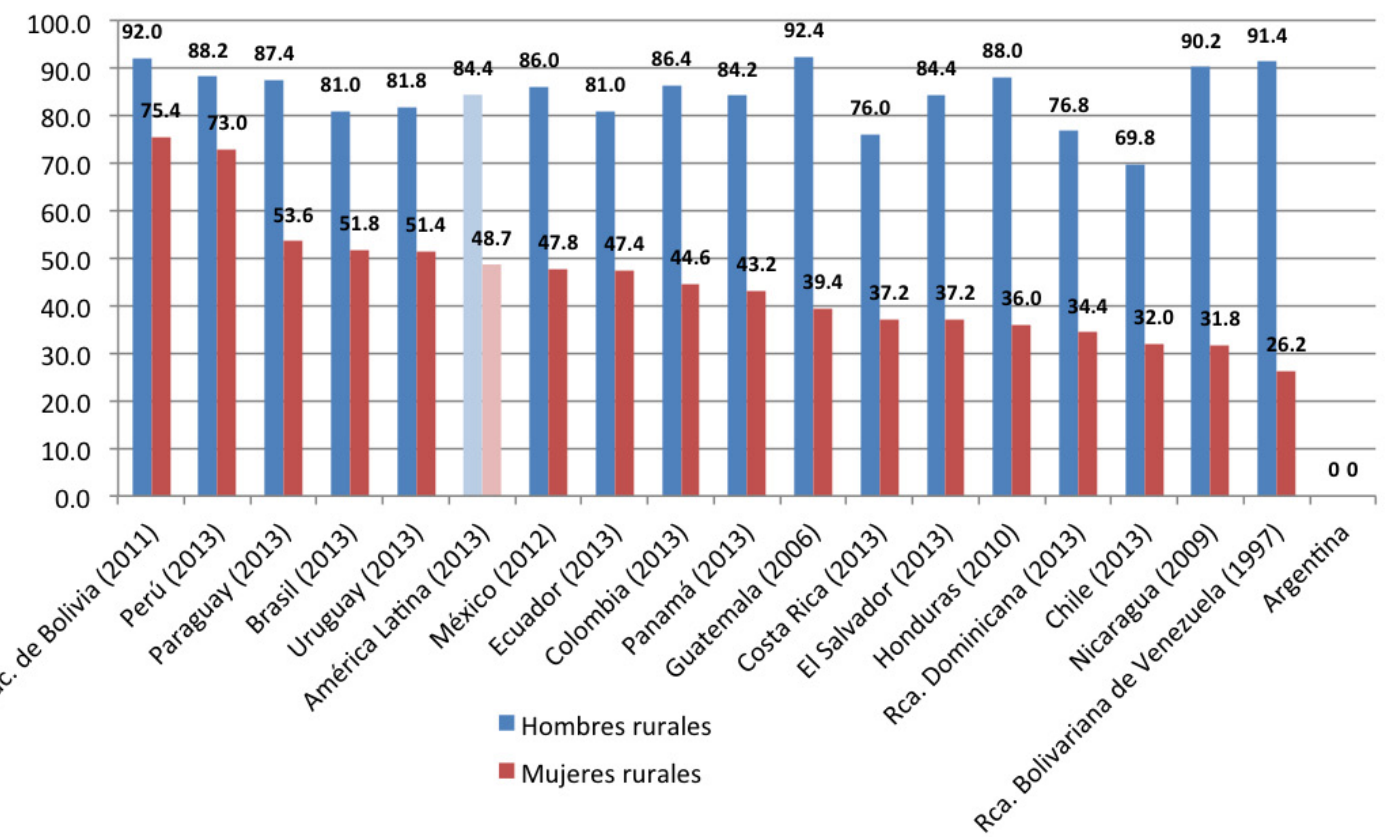

Dentro de las tasas mínimas de participación laboral femenina, se encuentra la mayoría de los países centroamericanos, con valores que oscilan entre el 40 y $31 \%$. El caso extremo en participación laboral femenina en América Latina (30,8\%), lo presenta Chile, coincidiendo con la tasa mínima de participación laboral masculina $(69,8 \%)$. Las mayores brechas entre tasa de participación laboral femenina y masculina lo tienen Nicaragua, Guatemala y Honduras (más de 50 puntos porcentuales de diferencia). En cambio, las menores brechas se encuentran en Perú y Bolivia, coincidiendo con las tasas más elevadas de participación laboral femenina (menos de 17 puntos porcentuales de diferencia). En todos los países latinoamericanos la tasa de participación laboral femenina no supera a la masculina.

Cuando se habla de empleo rural, se considera el lugar de residencia y no el domicilio laboral, por lo que erróneamente se presupone que se trata de actividades llevadas a cabo en zonas rurales. Según los datos estadísticos existentes no se puede determinar el número de habitantes rurales que trabajan en zonas urbanas ni el número de habitantes urbanos que trabajan en zonas rurales (Dirven, 2004:55). Dada esta imposibilidad, se interpretan todas las actividades económicas de la población rural como empleo rural, diferenciándolo en dos grupos laborales:

Empleo rural agrícola (ERA): actividades realizadas en el sector primario de la agricultura. La agricultura produce bienes agrícolas no procesados, utilizando los recursos naturales como uno de los factores de producción, y en los que el proceso puede ser de cultivo o de recolección.

Empleo rural no agrícola (ERNA): actividades distintas a las del sector agrícola primario. Desde el punto de vista sectorial, el ERNA puede considerarse como la suma del sector industrial más servicios desarrollados en el ámbito rural. 
La población rural ocupada latinoamericana se clasifica de acuerdo a alguna de las 16 grandes categorías indicadas en la Clasificación Internacional Industrial Uniforme (CIIU: 2009). Los sectores de agricultura, ganadería, caza y silvicultura, y pesca se agrupan bajo la categoría ERA y las 15 ramas restantes constituyen el ERNA: minería, manufactura, servicios básicos, construcción, comercio, hoteles y restaurantes, transporte y comunicaciones, intermediación financiera, servicios empresariales, administración pública, enseñanza, servicios sociales y de salud, servicio doméstico, otras actividades comunitarias y organizaciones extraterritoriales (Dirven y Köbrich, 2007:20).

Mujeres y hombres rurales se enfrentan a una desventaja estructural que limita sus opciones de empleo: la estructura productiva no crea suficientes empleos de buena calidad. Sumandose desventajas adicionales para las mujeres. En el campo es aún más rígida que en las urbes la asignación de roles que otorga a los hombres la principal responsabilidad en la producción y a las mujeres las restringe a la reproducción. Se las considera como trabajadoras secundarias cuya función es, en última instancia, complementar los ingresos del hogar o se las hace invisibles como trabajadoras familiares no remuneradas o como productoras para autoconsumo (OIT, 2012:52).

El ERA continúa siendo la principal actividad de la población rural en la mayoría de los países latinoamericanos. La gráfica 4, corresponden a 16 países latinoamericanos, el 65,3\% de los hombres rurales se dedica al sector primario, pero solo el 37,9\% de las mujeres rurales trabaja en este sector. En cambio en las mujeres rurales predomina el ERNA, representado por un $62,1 \%$. Estas cifras no incluyen algunos países altamente urbanizados como Argentina y Venezuela, ni países fuertemente ruralizados como Cuba y Haití, por lo que estas cifras promedio en Latinoamérica son aproximadas. Como se analizará posteriormente, debido a la poca información estadística con respecto a la distribución de propietarios de tierras agrícolas por sexo en los países latinoamericanos, no se puede contrastar la hipótesis sobre la existencia de una relación entre el predominio del ERNA femenino y las dificultades al acceso y control a la tierra por parte de las mujeres campesinas. Al ser este último muy precario (FAO, 1999:1), podría condicionar a las mujeres rurales a desempeñarse en el ERA.

En cambio, Bolivia, Perú, Ecuador y Brasil, más del 60 \% de las mujeres rurales se dedican a la agricultura (ERA). En Uruguay y Paraguay, es equitativa la dedicación de las mujeres rurales en ambos tipos de empleo. En el resto de los países latinoamericanos, la proporción de mujeres ocupadas en ERA es inferior al 36\%, tomando preponderancia el ERNA. Solo el 35,3\% de las mujeres colombianas trabaja en actividades del tipo ERA, por lo que el 64,7\% se dedica a ERNA. Luego le siguen Chile, Guatemala, Nicaragua, Panamá y Honduras, con mayoría de mujeres rurales que trabajan en puestos de trabajo en ERNA. En el caso extremo (El Salvador, México y Costa Rica) esta proporción de empleos femeninos en ERNA está por encima del 80\%, donde la República Dominicana presenta el mayor índice de mujeres rurales dedicadas a ERNA, 92,4\%. 
- Revista de Ciencias Sociales y Humanidades. ISSN-P: 0188-9834 ISSN-E: 2395-8669.

Gráfica 4. Proporción de población rural latinoamericana ocupada en Empleo Rural Agrícola (ERA) y Empleo Rural NO Agrícola (ERNA), segregada por sexo. 2012 (porcentaje)

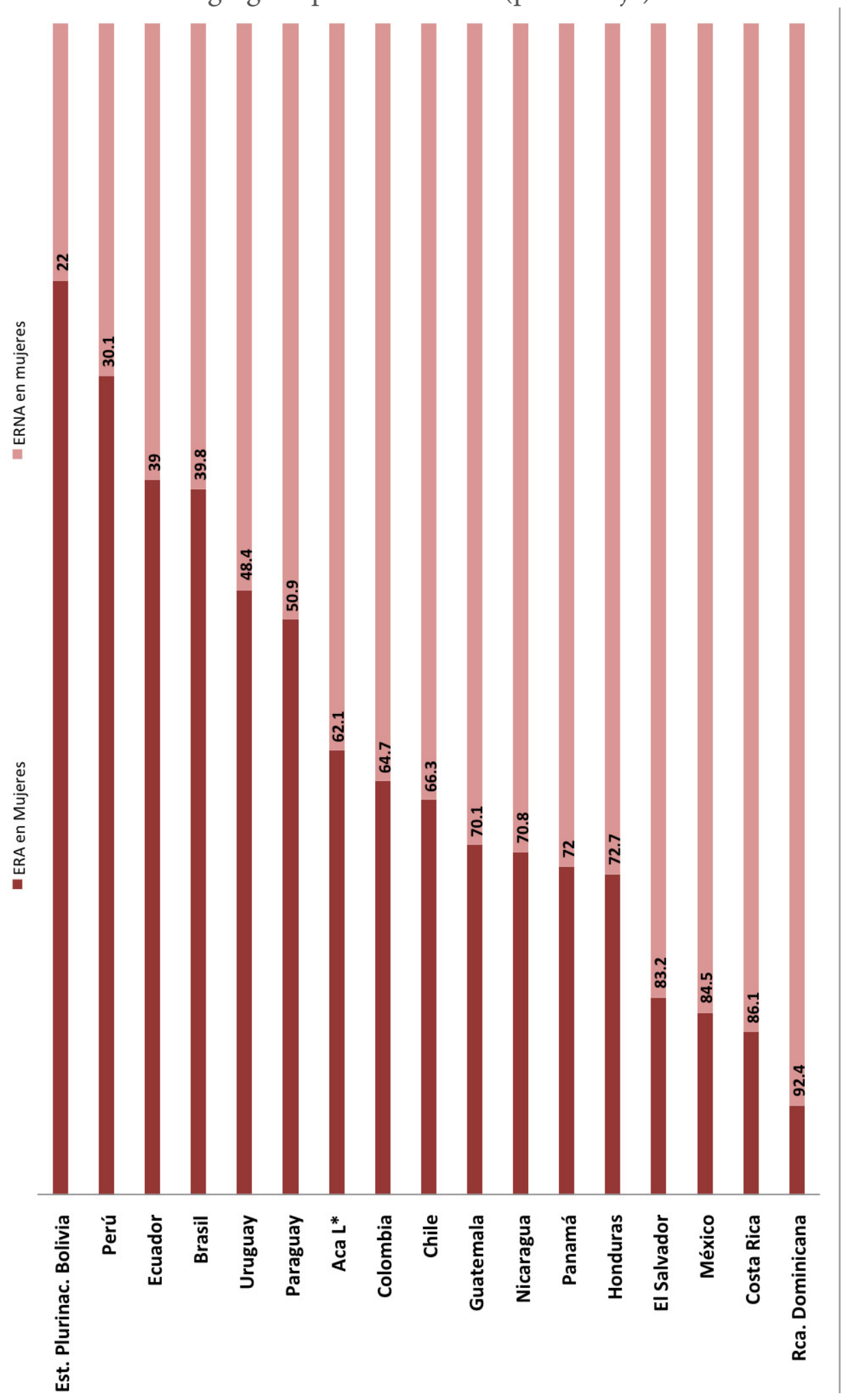


La mujer en el medio rural actúa en diversos ámbitos: como productora por cuenta propia (agricultura, alimentos, producción no agrícola), como trabajadora agrícola (mayoritariamente temporal) y rural no agrícola (comercio y servicios), y en tareas domésticas y productivas (cuidado de la huerta, del ganado, etc.) no remuneradas, lo que muchas veces resulta “invisible” para las estadísticas. Lamentablemente, las organizaciones públicas encargadas de los censos poblacionales en cada estado latinoamericano, no realizan una categorización de los puestos laborales en forma homogénea y específica en las zonas rurales. Sería interesante poder disponer de información según la categorización de los empleos rurales agrícolas para las mujeres propuesta por el Instituto Interamericano de Cooperación para la Agricultura (IICA, 2000:16). Esta institución presenta la categorización de los ERA para las mujeres rurales, según el tipo de organización y relaciones laborales, en cinco grupos: productoras agrícolas, cooperativistas agrícolas, comercializadoras agrícolas, jornaleras agrícolas y empleadas asalariadas.

Actualmente sólo se dispone de datos sobre la categorización ocupacional de la población económicamente activa en zonas rurales definida por la Organización Mundial del Trabajo (OIT, 1949:13), agrupada en cuatro categorías, empleadoras (patronas), asalariadas, trabajadoras por cuenta propia y trabajadoras familiares no remunerados (o fusionadas estas dos últimas).

En América Latina más del 58\% de las mujeres rurales ocupadas trabaja por cuenta propia o en trabajos familiares no remunerados (gráfica 5). Esta situación laboral se agrava en países como Bolivia y Perú, donde este porcentaje supera el 80\%. En el caso de Honduras, Guatemala, Colombia, Ecuador y Paraguay, el porcentaje de mujeres en esta situación supera más del 70\% de las mujeres rurales ocupadas. A nivel latinoamericano también se observa una brecha de género, del 8,3\%, entre el porcentaje de hombres ocupados que trabaja por cuenta propia o en trabajos familiares no remunerados y las mujeres en la misma situación. Pero esta brecha de género se dispara al 54,2\% en Guatemala. Existen casos opuestos a la tendencia general, Chile $(-4,3 \%)$, Nicaragua $(-5,3 \%)$ y República Dominicana $(-21,8 \%)$, donde la brecha de género en esta categoría ocupacional es negativa, es decir, existen más hombres rurales ocupados que trabaja por cuenta propia o en trabajos familiares no remunerados, que mujeres. 
- Revista de Ciencias Sociales y Humanidades. ISSN-P: 0188-9834 ISSN-E: 2395-8669.

Gráfica 5. Distribución de la población rural ocupada en América Latina, segregada por inserción laboral y sexo. 2012 (porcentaje)

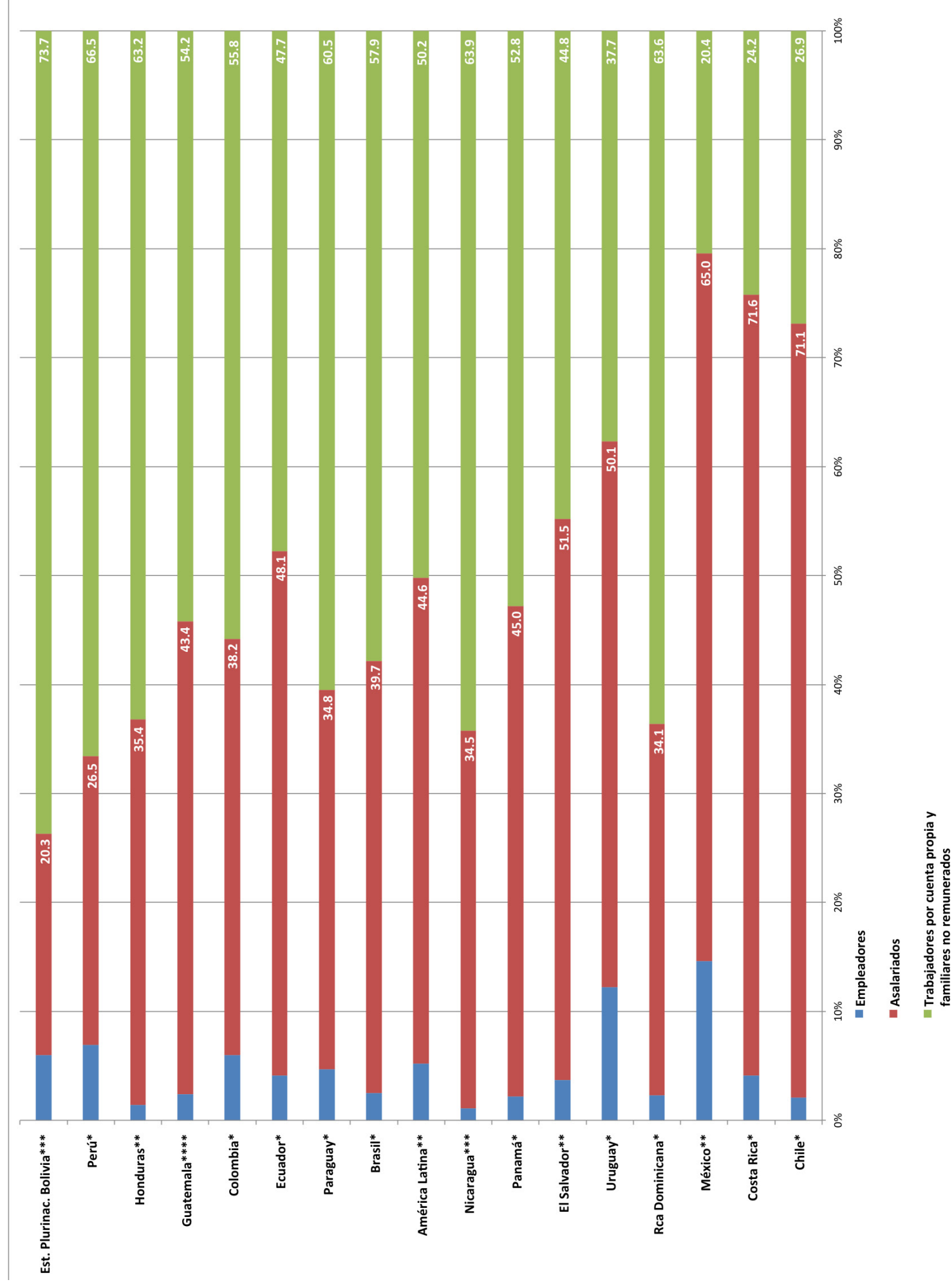


El 38,8\% de las mujeres rurales ocupadas son asalariadas. En esta categoría ocupacional se observa una gran heterogeneidad entre los distintos países latinoamericanos. Desde el caso de Bolivia, donde solo una pequeña fracción de las mujeres rurales ocupadas son asalariadas (10,3\%). En el caso opuesto se encuentra Chile, con casi el 76\% de las mujeres rurales ocupadas asalariadas e incluso presenta una brecha de género a favor de las mujeres de $-4,8 \%$. También presentan brecha de género negativa Nicaragua (-6\%) y República Dominicana $(-22,4 \%)$.

En Latinoamérica, el porcentaje de empleadores/ras o patrón/as es reducido. Las mujeres empleadoras que habitan en las zonas rurales de Guatemala, Paraguay, Brasil y Nicaragua presentan un porcentaje menor al $1 \%$. En cambio, cabe destacar México (12,5\%) y Uruguay (9\%), que presentan las tasas más elevadas de mujeres rurales empleadoras y significativamente presentan una brecha de género reducida que no supera el 3,2\%.

En el análisis de la tasa de participación laboral femenina rural se puede observar nuevamente una heterogeneidad entre los estados latinoamericanos. En promedio América latina presenta una tasa de participación laboral femenina del 45\%. Los países con mayores tasas de específica de participación laboral de la población rural femenina son, Bolivia y Perú, con más del 70\% de sus mujeres rurales dentro de la población económicamente activa. En contraste, Nicaragua y Chile, rondando apenas el $30 \%$ de sus mujeres rurales ocupadas. La única tendencia homogénea en el análisis de la tasa específica de participación laboral de la población rural latinoamericana, es que en todos los países, la tasa de participación laboral femenina nunca supera a la masculina. Resultados similares han sido presentados en el Panorama Laboral de 2012 de la OIT, aunque sus datos más actuales datan de 2010 (OIT, 2012:53).

La agricultura continúa siendo la principal actividad de la población rural en la mayoría de los países de América Latina. Pero en los últimos años, el ERNA está creciendo en importancia entre la población rural. Si los datos de población latinoamericana económicamente activa se segregan por sexo y se diferencia entre ERN y ERNA, más del 60\% de los hombres se dedican a ERN y no llega al $40 \%$ las mujeres dedicadas al sector primario. En cambio si se analiza a la población dedicada al ERNA, estas cifras se invierten, más del $60 \%$ de las mujeres rurales se dedican a ERNA y no llega al $40 \%$ los hombres dedicadas al mismo sector. El Panorama Laboral de 2012 de la OIT (OIT, 2012) obtienen resultados similares al evaluar el peso del ERA/ ERNA para mujeres rurales.

Los empleos del ERNA necesitan mano de obra calificada, por lo que se debe orientar parte de las ayudas públicas a la educación, formación laboral de población rural para que pueda acceder a este tipo de empleos, en especial a la mujer rural. Pero el último informe de la FAO sobre agendas políticas públicas hace un recorrido por las políticas y programas de los distintos ministerios y municipalidades, indica casi la total ausencia de menciones a la importancia del ERNA entre las ocupaciones principales de las mujeres rurales (FAO, 2013.c:34).

La mujer rural latinoamericana desempeña su actividad tanto en ERA como ERNA, casi el $60 \%$ trabaja por cuenta propia o en trabajos familiares no remunerados. Esta situación ubica a más de la mitad de las mujeres rurales latinoamericanas, cerca de 35 millones, en desventaja social porque no reciben un salario por sus actividades, generándoles dependencia económica respecto a sus familiares, e indirectamente de los hombres (Ballara y Parada, 2009:33). Sobre todo teniendo en cuenta que el grupo de trabajadoras familiares no remuneradas se encuentra subestimado en la mayoría de los estudios ya que un número importante de mujeres se auto clasifican como inactivas (no trabajan ni buscan trabajo); aunque efectivamente participan en las actividades de las explotaciones (FAO, 2013:2). 
A nivel latinoamericano, más del 38\% de las mujeres rurales ocupadas son asalariadas. La brecha de género en esta categoría ocupacional es de 5,8\%. Estos resultados coinciden con el panorama laboral presentado por la OIT en 2012 (OIT, 2012:54), que indica que sólo en Chile, Costa Rica, México y Uruguay las mujeres trabajan en mayor proporción como asalariadas, pero puntualiza que en estos países una minoría de las mujeres rurales se desempeña en la agricultura. (OIT, 2012:54). Por lo que nuevamente se indica que el ERNA es la fuente principal de empleos asalariados para las mujeres rurales.

\subsection{La legislación latinoamericana sobre la propiedad de tierras}

Numerosas instituciones internacionales insisten en promover la igualdad de los sexos y el empoderamiento de la mujer en las zonas rurales mundiales como solución integral a la pobreza. En las zonas rurales es prioritario fomentar el acceso equitativo a las tierras y el uso productivo de las mismas por parte de las mujeres. El acceso equitativo a las tierras se logra a través de reformas agrarias promulgadas por los Estados. Estas reformas agrarias deben tener en cuenta las cuestiones de género, impulsando los mercados y servicios de arriendo de tierra para las pequeñas agricultoras, la titulación conjunta de la tierra y bienes, y la revisión de leyes de herencia discriminatorias contra las mujeres.

La FAO expone desde el siglo pasado, que en América Latina el acceso y control a la tierra por parte de las mujeres campesinas es muy precario y la mayor parte de las reformas agrarias o las leyes de los países que regulan directa o indirectamente el acceso a la tierra discriminan a la mujer originado una situación de pobreza (FAO, 1999:1). El Programa de las Naciones Unidas para el Desarrollo (PNUD) sostiene que la igualdad del género tiene que ser parte de la estrategia de todos los países para erradicar la pobreza, como un fin y como un medio para acabar con otras formas de pobreza humana. Esto significa empoderar a la mujer garantizándole igualdad de derechos y acceso a la tierra, créditos y oportunidades de trabajo (PNUD, 1997:7). El informe sobre pobreza rural del Fondo Internacional de Desarrollo Agrícola (FIDA) reconoce que "reducir las barreras para que las mujeres controlen los bienes rurales, en particular la tierra, es crucial para establecer una política en contra de la pobreza" (FIDA, 2001:85). Es generalmente reconocido que, aunque el problema de la tierra afecta prácticamente a toda la población rural, las mujeres enfrentan mayor suma de dificultades. La brecha de género en la propiedad en América Latina es significativa y que ello se debe a cinco factores: preferencias masculinas en la herencia, privilegios masculinos en el matrimonio, sesgos masculinos en la comunidad y en los programas estatales en la distribución de la tierra y la desigualdad de género en el mercado de tierras. (Deere y Leon, 2003:925).

\section{Cuadro 3. Evolución de la legislación latinoamericana sobre} propiedad de tierras con aspectos de género.

Nivel 1. Legislación discriminatoria hacia la mujer con respecto a la posesión de tierras

República Dominicana
1962
Ley 5879/62 "Reforma Agraria" sólo da derecho de posesión de tierra a la esposa y no a la compañera. 


\begin{tabular}{|c|c|c|}
\hline Uruguay & 1948 & $\begin{array}{l}\text { Ley } 11029 \text { “Colonización” determina las condiciones para ser colonos, pero no se } \\
\text { menciona en forma explícita a la mujer. }\end{array}$ \\
\hline Argentina & 1958 & $\begin{array}{c}\text { Ley } 14451 \text { "Planes de Transformación Agraria” no menciona explícitamente a muje- } \\
\text { res en la compra con facilidades crediticias }\end{array}$ \\
\hline Chile & 1978 & $\begin{array}{c}\text { Ley 2247/78 "Regularización de la Tenencia de la Tierra", asignación individual de } \\
\text { tierras, sin mención explícitamente a la mujer. }\end{array}$ \\
\hline El Salvador & 1980 & $\begin{array}{l}\text { Decreto 153-154 “Ley Básica de la Reforma Agraria” no contempla específicamente } \\
\text { la propiedad de tierras de la mujer. }\end{array}$ \\
\hline \multicolumn{3}{|c|}{ Nivel 3. Legislación que sólo garantiza copropiedad de tierras dependiendo del estado civil } \\
\hline Argentina & 1980 & $\begin{array}{l}\text { Ley } 22298 \text { "Arrendamientos y aparcerías rurales" determina que por muerte del } \\
\text { arrendatario, continua su cónyuge. }\end{array}$ \\
\hline Costa Rica & 1990 & $\begin{array}{l}\text { Ley } 7142 / 90 \text { "Promoción de la Igualdad Social de la Mujer", derecho a la copropiedad } \\
\text { de la tierra a la mujer casada. }\end{array}$ \\
\hline $\begin{array}{l}\text { República } \\
\text { Dominicana }\end{array}$ & 1997 & $\begin{array}{c}\text { Ley } 55 / 97 \text { modifica a la Ley } 5879 / 62 \text {, incluye a compañera y sus hijos } \\
\text { Decreto } 144 / 98 \text { de titulación conjunta obligatoria de tierras adjudicadas aplicable a } \\
\text { parejas casadas o en uniones de hecho. }\end{array}$ \\
\hline Paraguay & 2002 & $\begin{array}{c}\text { Ley } 1863 \text { “Estatuto Agrario”, sólo garantiza la copropiedad por relación conyugal, } \\
\text { unión de hecho o herencia. }\end{array}$ \\
\hline \multicolumn{3}{|c|}{$\begin{array}{l}\text { Nivel 4. Legislación que garantiza propiedad de tierras por parte de la mujer independientemente de su estado } \\
\text { civil }\end{array}$} \\
\hline Cuba & 1959 & $\begin{array}{l}\text { "Ley de Reforma Agraria 1959", propiedad de tierras adjudicada a mujer u hombre } \\
\text { que la trabajan, sin distinción. }\end{array}$ \\
\hline Nicaragua & $\begin{array}{l}1981 \\
1986 \\
1995\end{array}$ & $\begin{array}{l}\text { Decreto 826/81, participación de mujeres en programas de reforma agraria al reco- } \\
\text { nocer igualdad de derechos sin distinción de género. } \\
\text { Ley 14/86 “Ley de Reforma Agraria" integra a la mujer en la selección de beneficia- } \\
\text { rios para la adjudicación de tierras. } \\
\text { Ley } 209 / 95 \text { “Estabilidad de la Propiedad”, titulación conjunta o mancomunada a } \\
\text { parejas. }\end{array}$ \\
\hline Ecuador & 1994 & $\begin{array}{l}\text { Ley } 54 \text { “Desarrollo Agrario", mercado libre de tierras, donde hombres y mujeres pue- } \\
\text { den poseer tierras sin distinción de su estado civil. }\end{array}$ \\
\hline Honduras & 2000 & $\begin{array}{l}\text { Decreto } 34 / 00 \text { “Ley de Igualdad de Oportunidades para la Mujer” en tenencia de } \\
\text { tierra, crédito y vivienda. } \\
\text { Decreto } 82 / 04 \text { propiedad de la tierra a personas con independencia de su estado } \\
\text { civil. }\end{array}$ \\
\hline Venezuela & 2001 & $\begin{array}{l}\text { Decreto } 1546 \text { “Ley de Tierras y Desarrollo Agrario", derecho de propiedad agraria a } \\
\text { campesinos y campesinas. }\end{array}$ \\
\hline
\end{tabular}




\begin{tabular}{|c|c|c|}
\hline Guatemala & 1962 & $\begin{array}{l}\text { Decreto } 1551 \text { “Ley de Transformación Agraria”, prioridad en el acceso a tierras a } \\
\text { familias de madres solteras y viudas. }\end{array}$ \\
\hline Brasil & 1973 & $\begin{array}{l}\text { Ley } 6001 / 73 \text { "Estatuto de Indio", titulación opcional de tierras a hombres y mujeres } \\
\text { con preferencia a mujeres jefas de hogar. }\end{array}$ \\
\hline México & $\begin{array}{l}1992 \\
1998 \\
2012\end{array}$ & $\begin{array}{l}\text { Ley Agraria de 1992, considera ejidarios tanto a hombres como mujeres., destinando } \\
\text { las mejores tierras colindantes con las zonas urbanas para explotación exclusiva de } \\
\text { mujeres mayores de } 16 \text { años. } \\
\text { Reglamento de Ley Agraria, fomenta la organización y desarrollo de la mujer campe- } \\
\text { sina. } \\
\text { Ley de Desarrollo Rural Sustentable, incluye programas de igualdad de género y para } \\
\text { la mujer, otorgando prioridad a sus unidades productivas. }\end{array}$ \\
\hline Colombia & 2003 & Decreto $2998 / 03$, beneficios a la mujer rural para la titularidad de tierras. \\
\hline Bolivia & 2006 & $\begin{array}{l}\text { Ley 3545/06 "Reconducción Comunitaria de la Reforma Agraria”, prioridad a la mu- } \\
\text { jer en saneamiento y distribución de tierras. }\end{array}$ \\
\hline
\end{tabular}

Fuente: Elaboración propia con datos de FAO (2014).

El cuadro 3 presenta la evolución temporal de la legislación latinoamericana sobre la propiedad de la tierra segregada por sexo y estado civil. Los países más avanzados con respecto a su legislación sobre la propiedad de tierras en función al género se pueden considerar a partir del nivel 4 (garantía de propiedad de tierras por parte de la mujer independientemente de su estado civil). Y como punto óptimo es el nivel 5 alcanzado por los países que prioriza a la mujer en el proceso de adquisición de tierras junto a otros beneficios.

Antes de la década de los 90, el caso más notorio de discriminación hacia la mujer con respecto a la tenencia, herencia o adquisición de tierras se daba en República Dominicana. Desde 1962 se discriminaba a la mujer no casada y a sus hijos ilegítimos privándola del derecho a la posesión de tierras. En otros países, no contemplaban en su legislación específicamente a la mujer, en lo referente a la condición de colono (Uruguay 1948) o posesión de tierras (Argentina 1958, Chile 1978, El Salvador 1980). En Argentina (1980) solo garantizaba la copropiedad de las tierras dependiendo del estado civil de las mujeres y solo por muerte del arrendatario.

En cambio, otros países garantizaban la copropiedad de las tierras por parte de la mujer independientemente de su estado civil. Cuba fue pionero en este tipo de legislación, en su reforma Agraria (1959) la propiedad de la tierra se adjudicaba a mujer u hombre que la trabajara. También Nicaragua (1981) decretó de participación de las mujeres en los programas de reforma agraria sin discriminación por género e integró a la mujer en la selección de beneficiarios para la adjudicación de tierras (1986).

Los países más avanzados en materia de igualdad de género en la propiedad de tierras en los 90', discriminaban positivamente a las mujeres más vulnerables. Otorgando prioridad en el acceso a tierras a familias de madres solteras y viudas (Guatemala 1962) y dando preferencia a mujeres indígenas jefas de hogar en la titulación de tierras (Brasil 1973). 
Según Deere (2001), el surgimiento y predominio de los gobiernos neoliberales en América Latina coincidieron con el crecimiento y la consolidación de dos nuevos movimientos sociales, el de mujeres y el indígena. Los dos movimientos impugnaron, la legislación agraria neoliberal que buscaba acabar con las reformas agrarias de los decenios anteriores.

La principal demanda del movimiento de las mujeres en cuanto a la tierra se ha centrado en el establecimiento de condiciones de igualdad entre hombres y mujeres, en especial mediante la adjudicación o titulación conjunta de tierras a parejas, independientemente de su estado civil, y/o la prioridad a las mujeres jefas de hogar.

A partir de 1990, quedan derogadas todas las leyes del tipo discriminatorio con respecto a la mujer según su estado civil. República Dominicana (1997) elimina la discriminación de la mujer no casada o a sus hijos en la posesión de tierras y otorga derechos a la compañera en la adjudicación de tierras, ratificando la titulación conjunta de tierras a parejas casadas o uniones de hecho.

En este nuevo período neoliberal, todas las legislaciones a nivel nacional, mencionan explícitamente a la mujer en temas de posesión de tierras. Salvo Uruguay, aunque se crea una Comisión Honoraria de la Mujer Rural (1996) para brindar un ámbito que asegure la interlocución con las mujeres del medio rural en el diseño y aplicación de políticas destinadas a ellas. En 2005, se pone en funcionamiento al Instituto Nacional de la Mujer, para promoción, diseño, coordinación, ejecución, seguimiento y evaluación de políticas públicas sobre género, y específicamente fortalecer su capacidad productiva mediante el acceso al empleo, crédito, tierra, tecnología e información.

Costa Rica (1990) otorga el derecho de la copropiedad a la mujer casada, pero en caso de unión de hecho otorga el título de propiedad a la mujer. Paraguay (2002) es el último país que aprueba una ley agraria donde sólo se garantiza la copropiedad por relación conyugal, unión de hecho o herencia. Estos dos países solo garantizan la copropiedad de tierras dependiendo del estado civil de la mujer. En cambio, los países que aprobaron legislación sobre la propiedad de tierras independientemente del estado civil de la mujer, se encuentra Ecuador (1994), Honduras (2000-2004) y Venezuela (2001). Honduras no solo decreta la igualdad de oportunidades para la mujer en materia de tenencia de tierras, sino también en créditos y vivienda, explícitamente indicando la independencia del estado civil.

En el escalón superior legislativo de igualdad de género sobre la posesión de tierras, se inicia en México, con leyes que priorizan a la mujer en el proceso de adquisición de tierras junto con otros beneficios. La ley agraria mexicana (1992) constituye unidades agrarias industriales para la mujer en las mejores tierras colindantes con las zonas urbanas. Luego, la reglamentación de la ley agraria (1998), se fomenta la organización desarrollo de la mujer campesina, y finalmente en 2012, reforma la ley de desarrollo rural sustentable, otorgando prioridad a las unidades productivas femeninas. Colombia continúa con el proceso legislativo igualitario y decreta beneficios a la mujer rural para la titularidad de tierras. Cerrando este ciclo legislativo, Bolivia (2006) garantiza y prioriza a la mujer en los procesos de saneamiento y distribución de tierras.

El análisis cuantitativo de la participación de la mujer rural en el acceso a las tierras se dificultad por la falta de datos primarios. Deere (2012) denuncia que es alarmante que en la nueva ronda de censos agropecuarios del siglo XXI en países de América Latina consideren quiénes son los propietarios de la tierra, fundamental para un análisis de la distribución de la propiedad de la tierra, tanto entre clases sociales como entre los géneros. La única herramienta actual disponible sobre la distribución de propietarios de 
tierras agrícolas por sexo está brindada por la base de datos de la FAO, pero con datos de pocos países latinoamericanos y disimiles temporalmente. Los datos disponibles (FAO, 2015:28) parecen indicar que las cuotas más altas de tenencia femenina de tierras (Nicaragua 20\%, México 32\% y Ecuador 51\%) coinciden con los países en nivel legislativo 4 y 5 . En Paraguay la cuota de tenencia femenina de tierras es alta (27\%), pero no está acompañada por una legislación avanzada sobre la propiedad de tierras en función al género (nivel 3- Legislación sólo garantiza la copropiedad de tierras dependiendo del estado civil). Finalmente Honduras, aunque cuenta con una legislación de nivel 4, la cuota de tenencia femenina de tierras es baja (14\%).

Es necesario garantizar el acceso de la mujer rural a la tierra por medio de la formulación de marcos jurídicos con perspectiva de género y la eliminación de los factores culturales e institucionales que impiden el reconocimiento de la mujer como productora. Las mujeres sólo suelen aparecer como responsables de una explotación agrícola cuando está ausente el hombre (debido a su migración) o cuando el hombre ha fallecido y ella ha recibido la tierra por herencia (Ballara y Parada, 2009:33).

\section{Conclusiones}

Los estudios sobre la población rural son de suma importancia como base para la elaboración de las políticas de ayudas a los distintos grupos poblacionales, en especial si se tratan de los grupos más vulnerables. Se hace necesaria la intervención de políticas que creen un nuevo concepto rural y no la limiten a un segundo plano de acción.

La heterogeneidad de la distribución de la población femenina rural latinoamericana se debe tener en cuenta para el desarrollo y aplicación de políticas públicas. Sería más efectiva la aplicación de políticas públicas a mujeres rurales en los pequeños países (centroamericanos), con un efecto importante en la economía global del país en cuestión, además del efecto directo sobre el colectivo de mujeres rurales. También se debe considerar que, a pesar de que las mujeres rurales en los países grandes el porcentaje es reducido, a nivel latinoamericano supone más de la mitad del total de mujeres rurales. En estos países, aunque el porcentaje de mujeres rurales no es relevante, la puesta en marcha de políticas públicas de ayuda a estas mujeres rurales supondría un gran efecto directo en este colectivo.

En todos los países existen brechas de género en la población económicamente activa, coincidiendo con la característica que son los países más ruralizados. Las políticas de desarrollo rural deberían eliminar el sesgo a favor del ERA y promover el desarrollo del ERNA, fortaleciéndolos a ambos. Sería prioritario que las agendas de políticas públicas de los países o regiones latinoamericanas consideren acciones y habiliten los canales financieros necesarios para el desarrollo del ERNA. No solo favorecerían el desarrollo rural en general sino también a las mujeres rurales ya que el ERNA es el sector que abarca las principales ocupaciones de las mujeres rurales latinoamericanas.

Los datos de categorización ocupacional en mujeres rurales son significativos para la elaboración de políticas para el desarrollo rural. La feminización de la fuerza de trabajo rural se refiere al aumento de presencia de las mujeres rurales en ERA y/o ERNA como empleadoras, asalariadas o trabajadoras por cuenta propia o trabajadoras familiares no remuneradas. Pero dependiendo de la categorización laboral a la que se incorporan las mujeres rurales ocupadas, se pueden producir distintos tipos de feminización 
de la fuerza de trabajo rural, totalmente opuestos entre sí y con consecuencias muy diferentes. Si la feminización se produce en la gestión de explotaciones agrícolas, es decir, aumenta el porcentaje de mujeres empleadoras o patronas, el efecto es positivo en este colectivo. En cambio si crece la feminización en la fuerza de trabajo rural informal, es decir, las mujeres rurales se emplean en puestos de trabajos informales no remunerados, esto tiene como consecuencias un aumento de la desigualdad de género y como consecuencia un aumento de pobreza femenina.

En los últimos decenios, la mayoría de países latinoamericanos han realizado modificaciones legislativas respecto al acceso a la tierra para una mejor igualdad de género. Pero el fin dista mucho de la realidad, ya que en el reconocimiento social de estos derechos aún persiste la inequidad. Sería necesario un esfuerzo mayor en el dictado de leyes con discriminación positiva hacia la mujer rural, no solamente en lo que respecta a la titularidad de tierras sino también el acceso a créditos, asistencia técnica y capacitación, a través de la planificación de políticas públicas agrarias con enfoque de género.

Las mujeres rurales son uno de los grupos sociales más vulnerables en el ámbito laboral latinoamericano, debido a su baja participación laboral, a la categorización ocupacional mayoritaria como trabajadoras por cuenta propia y familiar no remunerado. Pero sus posibilidades de mejorar su condición de vulnerabilidad social laboral al emplearse principalmente en el ERNA, con gran potencial en el desarrollo rural, es a través del apoyo legislativo para mejorar el acceso a los bienes de tierra. Esta medida podría aumentar su empleabilidad en el ERA a través de un aumento en la categorización a empresarias. El apoyo legislativo para mejorar el acceso a los bienes de tierra tienen que ir acompañado de políticas púbicas que tengan en cuenta la existencia de las mujeres rurales como trabajadoras en el ámbito rural y el diseño de medidas de ayuda especialmente dirigidas a este colectivo.

La sostenibilidad social y económica del medio rural necesita del arraigo femenino y por ello es imprescindible no sólo crear empleos, sino que las mujeres puedan acceder y permanecer en el mercado laboral con un buen grado de motivación y conciencia emprendedora, y por supuesto mejorar sus condiciones laborales. De esta manera puede acceder a oportunidades que le permitan salir de su condición de vulnerabilidad laboral y social.

\section{Referencias}

Ballara, Marcela y Soledad Parada. 2009. El empleo de las mujeres rurales. Lo que dicen las cifras. FAO/ CEPAL Santiago de Chile.1-96.

Ban Ki-moon. 2016. Conferencia sobre el desarrollo de los objetivos de desarrollo sostenible el día internacional de la mujer rural. Enlace disponible en: http://www.unwomen.org/es/news/in-focus/ruralwomen-food-poverty\#notes. Última consulta: 15 de junio de 2016.

CEPAL (Comisión Económica para América Latina y el Caribe). 2011. Anuario Estadístico de América Latina y el Caribe 2011. Santiago de Chile, 1-222.

CEPAL (Comisión Económica para América Latina y el Caribe). 2013. Panorama Social de América Latina 2013. Santiago de Chile, 1-228.

CIIU (Clasificación Industrial Internacional Uniforme). 2009. Clasificación Industrial Internacional Uniforme de todas las actividades económicas, Rev.4. Organización de Naciones Unidas, Departamento 
de Asuntos Económicos y Sociales División de Estadística. Informes estadísticos Serie M, No. 4/Rev. Enlace disponible: http://unstats.un.org/unsd/publication/seriesM/seriesm_4rev4s.pdf. Consultada 12 de junio de 2016.

Deere, Carmen Diana. 2001. Institutional Reform Of Agriculture Under Neoliberalism, The Impact Of The Women's And Indigenous Movements. Latin American Research Review, 36 (2):31-63.

Deere, Carmen Diana y Magdalena León. 2003. The Gender Asset Gap: Land in Latin America, World Development, 31 (6): 925-947.

DOI: http://dx.doi.org/10.1016/S0305-750X(03)00046-9.

Deere, Carmen Diana. 2012. Tierra y la autonomía económica de la mujer rural: avances y desafíos para la investigación. Revista ANTHROPOLÓGICAS, 23 (1):12-66.

Dirven Martine. 2004. El empleo rural no agrícola y la diversidad rural en América Latina. Revista CEPAL, 8:49-69.

Dirven Martine y Claus Köbrich. 2007. Características del empleo rural no agrícola en América Latina con énfasis en los servicios. Serie Desarrollo Productivo 174 CEPAL: 1-82.

Faiguenbaum, Sergio. 2011. Definiciones oficiales de "rural" y/o "urbano" en el mundo. En: Definiciones oficiales de "rural" y/o "urbano" en el mundo. Santiago de Chile: CEPAL, 67-107.

Faiguenbaum, Sergio. César Ortega y Fernando Soto Baquero. 2013. Pobreza rural y políticas públicas en América Latina y el Caribe. Roma: FAO, 1-292.

FAO (Organización de las Naciones Unidas para la Agricultura y la alimentación). 1999. El acceso de la mujer rural latinoamericana a la tierra. Roma: FAO, 1-4.

FAO (Organización de las Naciones Unidas para la Agricultura y la alimentación). 2013. Notas políticas sobre mujeres rurales. №2. Roma: FAO: 1-4.

FAO (Organización de las Naciones Unidas para la Agricultura y la alimentación). 2015. Gender and Land Statistics. Recent developments in FAO's Gender and Land Rights Database. Technical Note: 28.

FIDA (Fondo Internacional para el Desarrollo Agrícola). 2001. Rural Poverty Report 2001: The Challenge of Ending Rural Poverty: 1-266.

FPNU (Fondo de Población de las Naciones Unidas). 2010. América Latina: avances y desafíos de la implementación del Programa de Acción de El Cairo (2004-2009): 1-119.

IICA (Instituto Interamericano de Cooperación para la Agricultura). 2000. Situación de las mujeres rurales. En: Mujeres y Equidad de género en la nueva visión de la ruralidad. Capítulo III.: 15-34.

Kaztman, Rubén. 2000. Notas sobre la medición de la vulnerabilidad social. BID-Banco Mundial-CEPAL-IDEC, 5, 275-301.

ONU (Organización de las Naciones Unidas). 2012. La mujer rural y los Objetivos de Desarrollo del Milenio. FAO y FIDA: 1-12.

ONU Mujeres (2015). Enlace disponible: http://www.unwomen.org/es/news/in-focus/rural-womenfood-poverty. Consultada 12/06/2016.

OIT (Organización Mundial del Trabajo). 1949. VI Conferencia Internacional de Estadígrafos del Trabajo: 1-80.

OIT (Organización Mundial del Trabajo). 2012. El empleo de las mujeres rurales en América Latina. Panorama Laboral para América Latina y el Caribe 2012: 1-116.

OIT (Organización Mundial del Trabajo). 2013. Brechas entre el empleo rural y urbano. Panorama Labo- 
ral para América Latina y el Caribe 2013: 39-42.

Oxfam.2014. Mujeres rurales, indígenas y campesinas de América Latina: Una agenda frente al cambio climático: 3.

Pizarro, Roberto. 2001. La vulnerabilidad social y sus desafíos, una mirada desde América Latina. Serie estudios estadísticos y prospectivos. CEP: 1-69.

PNUD (Programa de las Naciones Unidas para El Desarrollo). 1997. Human Development Report 1997: $1-245$.

Quintanilla Barba, Carmen. 2002. Las mujeres rurales construyen su futuro. Libro blanco de la agricultura y el desarrollo rural: 1-9.

Rodríguez Vignoli, Jorge. 2001. Vulnerabilidad y grupos vulnerables: un marco de referencia conceptual mirando a los jóvenes. Serie Población y desarrollo CEPAL: 1-62.

Sabalain Cristina. 2011. Hacia una nueva definición de "rural" con fines estadísticos en América Latina. Capítulo XX de El concepto de "rural" en los países de la región. CEPAL: 21-29.

Weller, Jürgen. 2009. El fomento de la inserción laboral de grupos vulnerables. Consideraciones a partir de cinco estudios de caso nacionales: 1-123.

Weller, Jürgen. 2012. Vulnerabilidad, exclusión y calidad del empleo. Perspectiva latinoamericana. Revista Internacional de Estadística y Geografía, 3 (2): 82-97. 